Classification

Physics Abstracts

$64.70 \mathrm{~K}$

\title{
INFLUENCE DE LA TEMPÉRATURE SUR LES PRESSIONS DE TRANSITIONS DES HALOGÉNURES DU RUBIDIUM - DONNÉES THERMODYNAMIQUES
}

\author{
J. PEYRONNEAU et A. LACAM \\ Universités Paris VI \& VII, Institut de Physique du Globe, \\ Laboratoire de Géochimie et Cosmochimie *, Groupe de Géophysique sous Hautes \\ Pressions, 4, place Jussieu, Tour 14, 75230 Paris Cedex 05, France
}

(Reçu le 13 juillet 1977, révisé le 5 décembre 1977 et accepté le 6 décembre 1977)

\begin{abstract}
Résumé. - L'utilisation, sous haute pression hydrostatique, d'un four interne de grand volume, a permis de déterminer l'influence de la température sur les pressions de transitions des halogénures du rubidium. Les mesures volumétriques ont été effectuées à partir de la zone d'indifférence, ce qui a permis d'améliorer la précision des résultats par rapport à ceux précédemment publiés. Le coefficient de variation $\mathrm{d} P / \mathrm{d} T$ a été trouvé constant entre 22 et $440^{\circ} \mathrm{C}$. Les résultats expérimentaux ont été utilisés pour calculer les données thermodynamiques de ces transitions à $25^{\circ} \mathrm{C}$.

Abstract. - By using an internal heating device under purely hydrostatic conditions, the temperature dependence of the polymorphic transitions of the rubidium halides has been determined up to $440^{\circ} \mathrm{C}$. The volumetric measurements, carried out by measuring the width of the zone of indifference, have provided more accurate results than those previously published. The variation $\mathrm{d} P / \mathrm{d} T$ is constant between 22 and $440^{\circ} \mathrm{C}$. The experimental results have been used to calculate the thermodynamical data for these transitions at $25^{\circ} \mathrm{C}$.
\end{abstract}

1. Introduction. - $\mathrm{Si}$ les transitions polymorphiques des halogénures alcalins induites par la pression, ont fait l'objet de nombreux travaux tant expérimentaux que théoriques, en revanche, peu d'études ont été consacrées à l'influence de la température qui permet d'accéder aux propriétés thermodynamiques de ces réactions.

Les travaux les plus importants réalisés dans ce domaine sont attribuables en particulier à Bridgman [1], Pistorius [2, 3] et Darnell et McCollum [4]. Cependant leurs résultats expérimentaux présentent des divergences assez importantes, et les résultats de Pistorius, de Darnell correspondent à des domaines de températures disjoints. Enfin les techniques expérimentales utilisées ont limité la précision des résultats obtenus.

Lors de précédents travaux nous avons pu montrer que l'utilisation de la méthode dite de zone d'indifférence telle qu'elle a été définie par Bridgman [5] permettait, sous certaines conditions expérimentales, de déterminer la pression de transition sous haute pression avec une précision nettement supérieure à celle que l'on obtient en utilisant, comme l'ont fait les autres auteurs, le centre du cycle d'hystérésis.

\footnotetext{
* Laboratoire associé au C. N. R. S.-LA n 196
}

Le but du présent travail a été de déterminer par cette méthode l'influence de la température sur les pressions de transition des halogénures de rubidium à l'exception du fluorure. Les expériences ont été effectuées à des températures comprises entre $20^{\circ} \mathrm{C}$ et $440^{\circ} \mathrm{C}$, c'est-à-dire dans un domaine recoupant les différentes données précédemment publiées.

L'utilisation d'un four interne de grand volume nous a permis d'utiliser la méthode volumétrique propre à la détermination de la zone d'indifférence, et ceci dans un milieu compresseur liquide parfaitement hydrostatique dans le domaine de pression considéré.

Les résultats préalablement obtenus sur ces corps et la connaissance précise de la pression et de sa variation $\mathrm{d} P / \mathrm{d} T$ en fonction de la température nous ont permis de calculer les propriétés thermodynamiques des transitions étudiées.

2. Conditions expérimentales. - 2.1. HAUTES PRESsions. - L'appareil haute pression est un dispositif classique piston-cylindre, fonctionnant en milieu compresseur liquide, qui a été précédemment décrit $[7,8]$.

Huit entrées de courant étanches permettent des mesures de pression et de température à l'intérieur de l'enceinte expérimentale et l'alimentation du four interne. 
A la température ambiante les mesures de pression sont effectuées par l'intermédiaire de jauges résistantes en manganine. Lorsque la température s'élève, la résistance de la manganine varie d'une façon non linéaire et son coefficient de pression n'est pas constant. Il a donc été nécessaire d'adapter à l'appareillage un système de mesure de la pression qui soit indépendant de la température intérieure de l'enceinte haute pression.

Dans ce but, nous avons utilisé des jauges de contraintes fixées sur le poussoir moyen de la presse qui n'est en aucun cas perturbé par les variations thermiques internes. Les quatre jauges de contraintes, montées en pont de Wheastone fournissent une tension proportionnelle à la poussée exercée sur leur support. Les jauges sont alimentées par un pont d'extensiométrie à fréquence porteuse de $5 \mathrm{kHz}$ qui sert également de détecteur. Le système de mesure ainsi constitué a été étalonné à la température ambiante par rapport à une jauge de manganine placée directement dans le liquide compresseur. La réponse a été trouvée linéaire et reproductible par rapport à la pression réelle dans la chambre expérimentale. Ce dispositif s'est révélé d'autre part très sensible et une variation de pression de quelques bars est très facilement mesurable.

Il faut remarquer toutefois que les variations de tension à la sortie du pont ne traduisent que la poussée exercée sur le piston mobile de la chambre. Il faut ajouter à cette dernière les frottements des joints du piston mobile sur les parois de l'enceinte. Avec le dispositif d'étanchéité utilisé, ils représentaient environ $10 \%$ de la pression réelle.

Les frottements étant de sens opposés à la compression et à la décompression, on obtient deux droites d'étalonnage distinctes qui permettent de déduire les pressions réelles.

Avec une jauge manganine référence préalablement étalonnée on peut atteindre par cette technique une précision de l'ordre de $0,5 \%$ sur la mesure de la pression tout en s'affranchissant totalement de la température expérimentale.

2.2 Hautes températures. - Le chauffage de l'échantillon s'effectue par l'intermédiaire d'un four interne situé dans le liquide transmetteur de pression. La figure 1 montre le schéma du dispositif utilisé. L'élément chauffant est un enroulement d'un fil de $\mathrm{Ni}$. Cr de 0,2 $\mathrm{mm}$ de diamètre. La résistance ainsi constituée est de l'ordre de $100 \Omega$. L'enroulement est placé entre deux chemises cylindriques de pyrophyllite. Ce corps porté à haute température subit une dilatation non réversible. Ainsi en ajustant correctement la chemise intérieure supportant l'élément chauffant dans une chemise extérieure préalablement déformée, on obtient en chauffant l'ensemble un sertissage des deux cylindres. Celui-ci assure à la fois un bon isolement électrique dans l'enroulement et un contact intime avec le support transmetteur de chaleur.

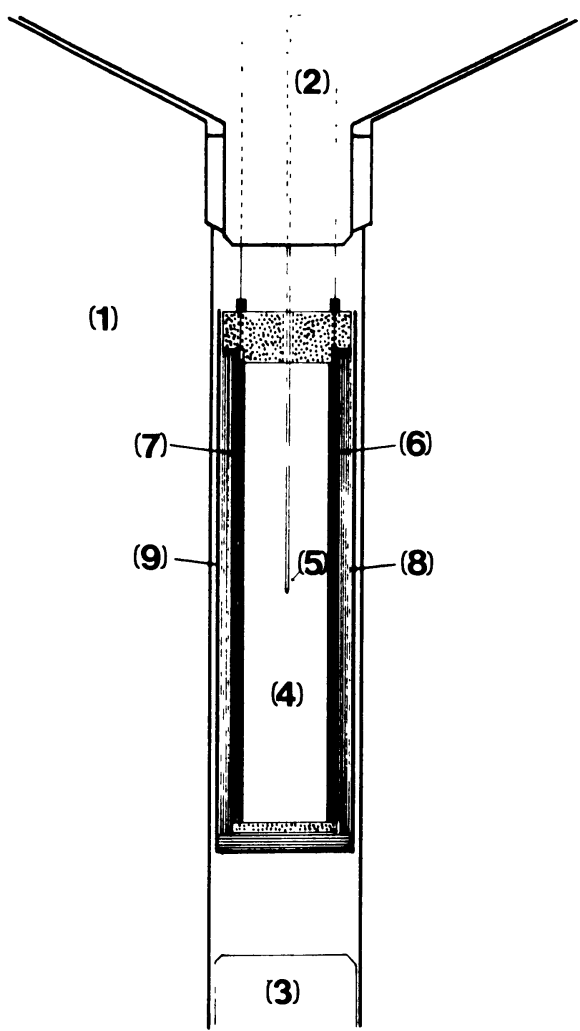

FIG. 1. - Dispositif de chauffage : 1 . enceinte haute pression ; 2. obturateur fixe; 3. piston mobile ; 4. échantillon ; 5 . thermocouple ; 6. pyrophyllite ; 7. enroulement chauffant ; 8 . isolement ; 9. laiton.

[Internal heating device : 1 . high pressure cell ; 2 . closure; 3. piston ; 4. sample ; 5. thermocouple; 6. pyrophyllite ; 7 . heating coil ; 8. insulation; 9 . brass.]

L'isolement thermique est réalisé à l'aide de chemises de nickel séparées les unes des autres par le liquide ambiant. Des picots maintiennent entre elles un écartement constant de l'ordre de $0,1 \mathrm{~mm}$. On obtient ainsi une série de couches successives métalliques et liquides présentant alternativement des conductibilités thermiques très différentes. Au niveau de chaque chemise de nickel qui constitue une discontinuité de conduction une partie de la chaleur est renvoyée par rayonnement vers l'intérieur du four. D'autre part les couches métalliques successives contribuent à diminuer les pertes thermiques en évitant les mouvements de conviction du liquide. Ces divers points peuvent expliquer l'efficacité de ce type d'isolement qui nous a permis d'atteindre sous pression des températures élevées avec une puissance d'alimentation relativement faible comme le montre la figure 2 .

Le four est alimenté par l'intermédiaire de deux thermocoax monofilaires de cuivre qui traversent l'obturateur fixe de la chambre. Les liaisons entre le fil chauffant et les fils d'alimentation sont assurées par des brasures.

Le diamètre de la partie utile du four est de $12 \mathrm{~mm}$, sa longueur de $55 \mathrm{~mm}$. Ces dimensions offrent un 


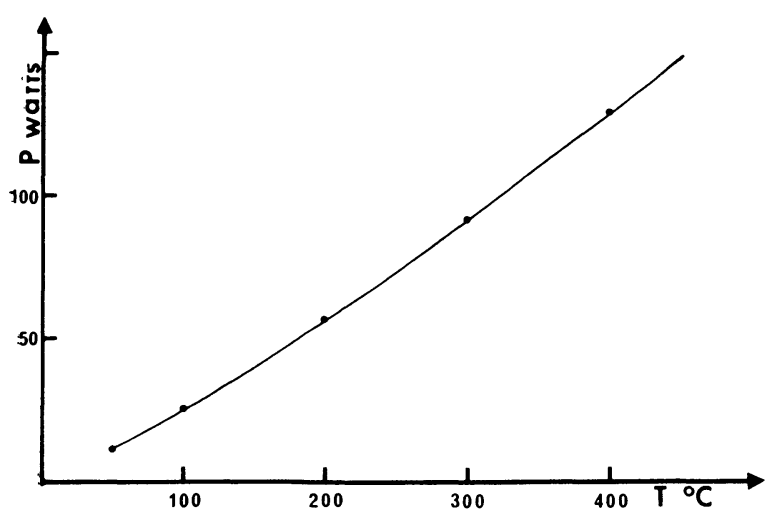

Fig. 2. - Puissance dissipée par le four interne.

[Power versus temperature in the furnace.]

volume libre pour l'échantillon de l'ordre de $6 \mathrm{~cm}^{3}$ ce qui est tout à fait suffisant pour réaliser des mesures volumétriques dans nos conditions expérimentales.

La température est mesurée et régulée à partir de la réponse d'un thermocouple chromel-alumel dont la soudure chaude est située à l'intérieur du four et qui traverse l'obturateur fixe dans une gaine thermocoax bifilaire. La régulation thermique du four est effectuées à l'aide d'un régulateur automatique direct MECI $06 \mathrm{AD}$. Le courant de sortie du régulateur, proportionnel à l'écart entre la température mesurée et la température consignée commande un amplificateur magnétique qui alimente une self à saturation variable. Ce dispositif a permis de réguler la température à l'intérieur du four à mieux de $0,1^{\circ} \mathrm{C}$ près. Compte tenu des pressions maximales atteintes, leur influence sur la réponse du thermocouple est négligeable et il n'a pas été fait de correction dans ce sens.

2.3 Transmetteur de pression. - Pour les expériences sur les halogénures de rubidium nous avons utilisé comme liquide transmetteur un mélange d'huile de SILICONE SI 550 et de n-pentane. L'huile de silicone SI 550 a une très bonne tenue thermique mais devient très visqueuse à température ambiante pour des pressions de 0,3 GPa [9]. L'addition de n-pentane permet d'élever la pression critique à température ambiante et de conserver l'hydrostaticité du milieu. La concentration en pentane est fonction de la pression de transition étudiée. Dans tous les cas il a une très bonne tenue thermique sous pression et aucune détérioration n'a été constatée pour des températures allant jusqu'à $440^{\circ} \mathrm{C}$.

2.4 Echantillons. - Tous les échantillons ont été utilisés sous forme d'une poudre placée directement à l'intérieur du four et occupant tout le volume utile.

Les produits de provenance Mercks (pureté 99,95\%) ont été préalablement recristallisés à partir d'une solution aqueuse saturée à chaud puis refroidie. Ils sont avant l'expérience desséchés sous une lampe infra-rouge. Les échantillons ainsi obtenus conduisent à des cycles d'hystérésis plus étroits qu'avec les produits bruts, ce qui est une condition particulièrement favorable à l'emploi de la méthode volumétrique et à la détermination de la zone d'indifférence.

3. Résultats expérimentaux. - Les pressions de transition ont été déterminées entre la température ambiante et $440^{\circ} \mathrm{C}$. Les mesures ont été effectuées à des intervalles réguliers de $30^{\circ} \mathrm{C}$. Pour chaque expérience on a tracé le cycle d'hystérésis par la méthode de discontinuité volumétrique préalablement décrite [7]. De même la zone d'indifférence a été déterminée dans tous les cas où elle pouvait fournir des résultats plus significatifs que le cycle; nous avons alors employé la méthode des paliers à volume total constant [6]. La figure $\mathrm{n}^{\circ} 3$ montre à titre d'exemple le diagramme expérimental obtenu pour $\mathrm{RbI}$ à $140^{\circ} \mathrm{C}$. La zone d'indifférence est indiquée par des flèches.

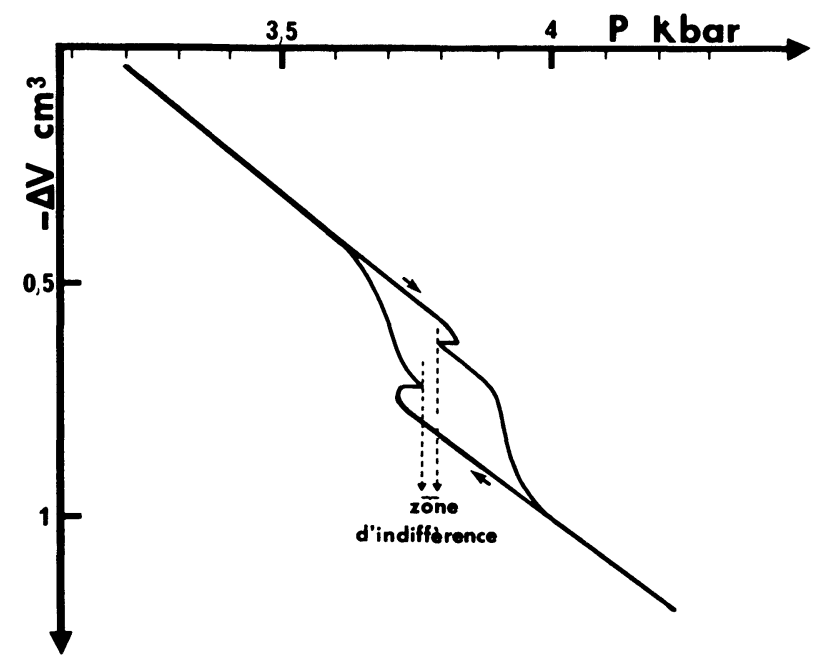

Fig. 3. - Diagramme expérimental de RbI à $140^{\circ} \mathrm{C}$.

[Experimental diagram for $\mathrm{RbI}$ at $140^{\circ} \mathrm{C}$.]

Le tableau I résume les résultats expérimentaux obtenus pour les trois corps. Les valeurs à $22^{\circ} \mathrm{C}$ sont celles préalablement obtenues sur un échantillonnage important de mesures qui avait fait l'objet d'un calcul statistique [10].

Les valeurs affectées d'un astérisque correspondent au centre du cycle d'hystérésis. Ce dernier a été pris en considération pour les températures les plus élevées. En effet pour des températures de l'ordre de $350^{\circ} \mathrm{C}$ le cycle devient étroit, la nucléation de la phase nouvelle a lieu près de l'équilibre thermodynamique et permet de le définir plus correctement que la zone d'indifférence dont la détermination est alors génée à la fois par la lenteur et le manque d'amplitude de la réaction.

Les autres valeurs consignées dans le tableau I correspondent au centre de la zone d'indifférence. Les incertitudes accompagnant les pressions de transition indiquent selon le cas la demi-largeur du cycle d'hystérésis ou de la zone d'indifférence obtenus. 


\section{TABleau I}

Résultats des mesures des pressions de transition entre $22^{\circ}$ et $440^{\circ} \mathrm{C}$ (en kbar)

[Results of the transition pressures measurements between $22^{\circ}$ and $440{ }^{\circ} \mathrm{C}$ (in kbar)]

$\begin{array}{rccc}T^{\circ} \mathrm{C} & \mathrm{RbI} & \mathrm{RbBr} & \mathrm{RbCl} \\ \overline{22} & 3,560 \pm 0,013 & 4,040 \pm 0,020 & 5,200 \pm 0,048 \\ 50 & 3,615 \pm 0,011 & 4,090 \pm 0,024 & 5,295 \pm 0,057 \\ 80 & 3,670 \pm 0,011 & 4,145 \pm 0,024 & 5,399 \pm 0,072 \\ 100 & 3,723 \pm 0,007 & 4,200 \pm 0,027 & 5,505 \pm 0,169 \\ 140 & 3,774 \pm 0,013 & 4,270 \pm 0,104 & 5,614 \pm 0,224 \\ 170 & 3,828 \pm 0,027 & 4,330 \pm 0,140 & 5,670 \pm 0,275 \\ 200 & 3,885 \pm 0,059 & 4,390 \pm 0,150 & 5,765 \pm 0,275 \\ 230 & 3,917 \pm 0,076 & 4,455 \pm 0,155 & 5,900 \pm 0,275 \\ 260 & 3,971 \pm 0,097 & 4,515 \pm 0,172 & 6,008 \pm 0,275 \\ 290 & 4,011 \pm 0,110 & 4,580 \pm 0,172 & 6,150 \pm 0,275 \\ 320 & 4,058 \pm 0,107 & 4,692 \pm 0,155 & 6,228 \pm 0,275 \\ 350 & 4,126 \pm 0,069 & 4,715 \pm 0,112 & * 6,322 \pm 0,275 \\ 380 & 4,183 \pm 0,058 & * 4,780 \pm 0,096 & * 6,410 \pm 0,220 \\ 410 & * 4,234 \pm 0,041 & * 4,845 \pm 0,069 & * 6,504 \pm 0,163 \\ 440 & * 4,289 \pm 0,013 & * 4,900 \pm 0,041 & * 6,592 \pm 0,075\end{array}$

Les résultats du tableau I ont été traités par la méthode des moindres carrés dans le but de déterminer la meilleure droite :

$$
P_{\mathrm{Tr}}=a+b T
$$

$P_{\mathrm{Tr}}$ est la pression de transition à la température $T$. $a$ est la pression de transition à $0^{\circ} \mathrm{C}$. $b$ est la variation $d P / d T$ de cette pression en fonction de la température.

Le tableau II donne les valeurs de $a$ et $b$ ainsi que celles publiées par d'autres auteurs ayant utilisé la même méthode numérique de calcul. Les domaines de température explorés dans chacun des cas sont également indiqués. Les incertitudes accompagnant nos résultats ont été évaluées compte tenu de nos marges d'erreurs expérimentales et conformément à la loi de propagation des erreurs à caractère aléatoire pour les droites de régression. Les déviations moyennes des points expérimentaux par rapport aux droites calculées sont de 8 bars pour RbI et $\mathrm{RbBr}, 14$ bars pour $\mathrm{RbCl}$; ces valeurs faibles confirment la bonne linéarité du phénomène étudié.

La figure 4 montre pour les trois corps étudiés les points expérimentaux obtenus ainsi que l'incertitude

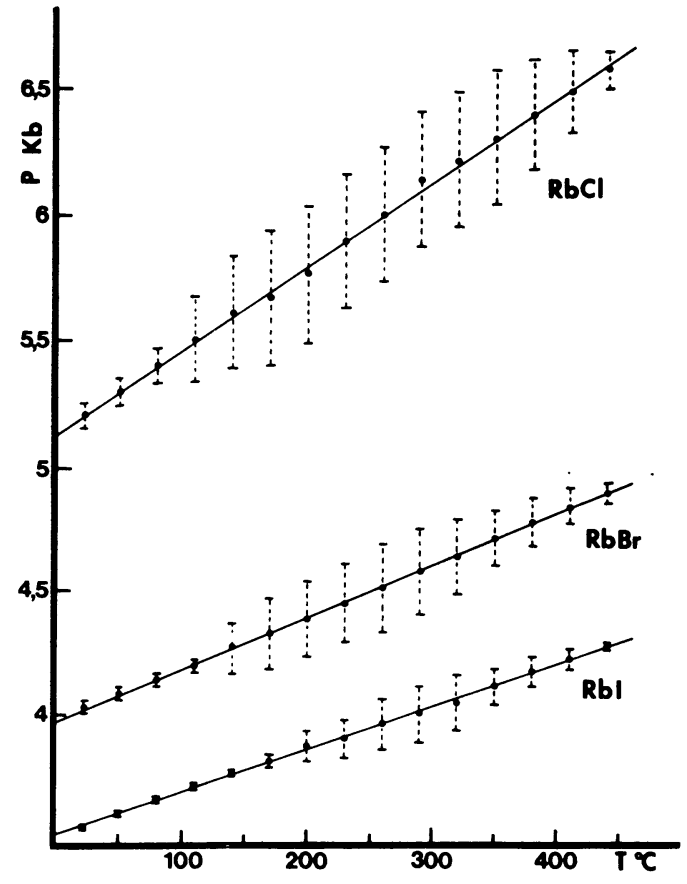

Fig. 4. - Pressions de transition en fonction de la température. [Transitions pressures versus temperature.]

sur chaque détermination de la pression de transition. Dans les trois cas la droite tracée est celle qui a été calculée par la méthode des moindres carrés.

Les valeurs nulles fournies pour $b$ par Bridgman sont attribuables au manque de précision de la méthode utilisée dans la gamme de température explorée. En effet Bridgman a fait varier la température entre 0 et $100^{\circ} \mathrm{C}$, domaine où l'effet d'hystérésis est important. Il a pris comme repère de la transition le centre du cycle d'hystérésis. L'incertitude sur chaque mesure est donc grande. De plus ses déterminations ont été gênées par la difficulté de situer avec précision le début des transitions en raison des vitesses de réactions faibles. Ainsi l'utilisation du cycle d'hystérésis dans un domaine de température défavorable et trop limité ne lui a pas permis d'obtenir des résultats significatifs.

Ces différents critères (largeur du cycle et imprécision dans la détermination du début des transitions)

\section{TABLEAU II}

Coefficients $a$ et $b$ de l'équation $P_{\mathrm{T} \mathrm{R}}=a+b T\left(a\right.$ en kbar, $b$ en kbar. $\left.{ }^{\circ} C^{-1}\right)$

[Values of the constants $\mathrm{a}$ and $\mathrm{b}$ in the equation $\mathrm{P}_{\mathrm{TR}}=\mathrm{a}+\mathrm{bT}\left(\mathrm{a}\right.$ in $\mathrm{kbar}, \mathrm{b}$ in $\left.\mathrm{kbar} .{ }^{\circ} \mathrm{C}^{-1}\right)$ ]

\begin{tabular}{lcccccc} 
& \multicolumn{2}{c}{$\mathrm{RbI}$} & $b \times 10^{3}$ & $a$ & $\mathrm{RbBr}$ & \multicolumn{2}{c}{$\mathrm{RbCl}$} \\
Bridgman & $4, \bar{c}$ & $\overline{0}$ & 4,60 & $\overline{0}$ & 5,00 & $\bar{b}$ \\
Pistorius & 3,54 & $1,94 \pm 0,4$ & 4,14 & $2,19 \pm 0,8$ & 5,20 & 3,01 \\
Darnell & 3,64 & $1,4 \pm 0,115$ & 4,88 & $1,371 \pm 0,124$ & 5,61 & $2,800 \pm 0,296$ \\
Ce travail & 3,530 & $1,71 \pm 0,12$ & 3,977 & $2,11 \pm 0,20$ & 5,125 & $3,38 \pm 0,40$
\end{tabular}


nous ont conduits à exploiter plus particulièrement la zone d'indifférence au cours de ce travail. En effet celle-ci est toujours plus étroite que le cycle jusqu'à $300^{\circ} \mathrm{C}$ et son centre correspond mieux à la pression d'équilibre thermodynamique puisqu'il représente un équilibre statique des deux phases. Enfin les limites de la zone d'indifférence peuvent être déterminées sans ambiguité avec une bonne précision.

La figure 5 met en évidence les avantages de l'utilisation de cette méthode. On constate d'abord que les

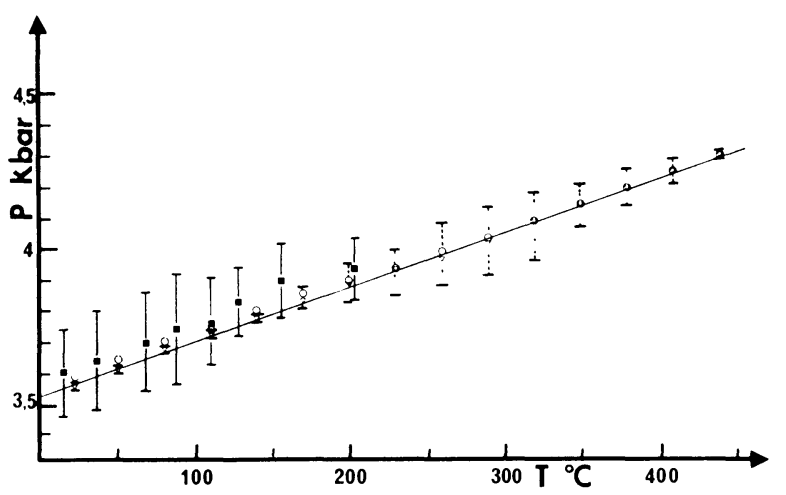

Fig. 5. - RbI- $:$ Pistorius- $\nabla$ : ce travail, zone d'indifférence$\bigcirc:$ ce travail, cycle d'hystérésis.

[RbI- $\square$ : Pistorius- $\nabla:$ this work, zone of indifference- $\bigcirc$ : this work, hysteresis loop.]

points correspondant au centre du cycle d'hystérésis * sont, jusqu'à des températures de l'ordre de $150^{\circ} \mathrm{C}$, extérieurs à la zone d'indifférence. Cela signifie que le cycle ne donne pas des résultats corrects dans cette gamme de température. A partir de $200^{\circ} \mathrm{C}$ la zone d'indifférence présente un élargissement significatif lié à un élargissement du cycle d'hystérésis et une diminution des vitesses de réaction. Mais on observe alors une bonne concordance entre les valeurs fournies par le cycle et par la zone d'indifférence. Il semble donc que pour des températures supérieures à $200^{\circ} \mathrm{C}$ le cycle d'hystérésis puisse fournir des résultats corrects pour la mesure des transitions. Ceci a été confirmé par ailleurs par Darnell et Mc Collum [4] qui considèrent n'avoir obtenus des résultats valables qu'au-dessus de $200^{\circ} \mathrm{C}$.

Enfin on a placé sur la figure 5 les points expérimentaux de Pistorius [3]. On voit aisément, compte tenu du rapport des domaines d'incertitudes, l'intérêt d'utiliser la zone d'indifférence dans la gamme de températures qu'il a étudiée. On peut noter aussi que, comme dans nos expériences, les points qu'il a obtenus se situent systématiquement à des pressions supérieures à ceux donnés par la zone d'indifférence. Ceci traduit le fait que, la surpression, écart entre la pression de nucléation et la pression d'équilibre thermodynamique, est fonction du sens de la réaction. Elle est dans le cas présent plus importante à la compression qu'à la décompression.
Lors de précédents travaux [7] nous avons constaté que ce phénomène était général et que la surpression nécessaire à la nucléation de la phase haute pression pouvait varier, en fonction des conditions expérimentales, dans des proportions beaucoup plus importantes que celles nécessaires à la nucléation de la phase basse pression. Le cycle variant ainsi d'une façon dissymétrique, son centre n'est donc pas un point fixe, mais fonction de paramètres expérimentaux tels que le nombre de cycles effectués, vitesse de compression, traitements préalables subis par l'échantillon, etc...

Par contre la zone d'indifférence donne des valeurs très reproductibles qui ne semblent pas affectés par ces divers paramètres [6].

Il faut noter enfin que les résultats pour $\mathrm{RbBr}$ et $\mathrm{RbCl}$ sont semblables à ceux qu'illustrent la figure 5 pour $\mathrm{RbI}$.

A l'examen du tableau II, on constate une assez grande divergence entre nos résultats et ceux de Darnell et Mc Collum. Ceci peut s'expliquer par le fait que ces derniers ont utilisé un milieu compresseur solide non hydrostatique. Dans ces conditions, la mesure des pressions est beaucoup plus aléatoire, et on introduit d'autre part des contraintes importantes dans l'échantillon. Nos résultats sont en meilleur accord avec ceux de Pistorius qui a utilisé un appareil haute pression à piston tournant plus proche de nos propres conditions expérimentales.

La connaissance de la variation $d P / d T$ de la pression de transition en fonction de la température permet de calculer les variations d'énergie interne $\Delta E$ et d'entropie $\Delta S$ pendant la transition, ainsi que la chaleur latente de transformation $L$. En effet en appliquant l'équation de Clausius-Clapeyron aux équilibres polymorphiques nous avons :

$$
\frac{\mathrm{d} P}{\mathrm{~d} T}=\frac{L}{T \Delta V}=\frac{\Delta S}{\Delta V}
$$

$\Delta V$, variation de volume accompagnant la transition, a été préalablement déterminée à la température ambiante [10].

A l'équilibre thermodynamique des phases, nous savons que les énergies libres des deux phases sont égales et la variation d'enthalpie libre de réaction est nulle soit:

$$
G=\Delta E-T \Delta S+P \Delta V=0 .
$$

Les différentes données thermodynamiques calculées par l'intermédiaire des équations précédentes et à partir de nos résultats expérimentaux sont consignées dans les tableaux III, IV et V, dans lesquels figurent par ailleurs les valeurs fournies par d'autres auteurs. La variation de volume du solide lors de la transition n'ayant été déterminée qu'à la température ambiante, les propriétés thermodynamiques que nous avons calculées sont valables pour les transitions polymorphiques des trois corps considérés à $25^{\circ} \mathrm{C}$. 


\section{TALBeAu III}

Données thermodynamiques pour la transition de $\mathrm{RbI}$ à $25^{\circ} \mathrm{C}$

[Thermodynamical data for the RbI transition at $25^{\circ} \mathrm{C}$ ]

Pistorius

Weir et al.

Darnell, Mc Collum

Nos résultats

$$
\begin{gathered}
\mathrm{d} P / \mathrm{d} T \\
\mathrm{kbar} /{ }^{\circ} \mathrm{C}
\end{gathered}
$$$$
1,94 \pm 0,4
$$

$$
\begin{gathered}
1,4 \\
1,7 \pm 0,12 \\
\\
\text { Thermodynam } \\
\mathrm{d} P / \mathrm{d} T \\
\mathrm{kbar} /{ }^{\circ} \mathrm{C} \times 10^{3}
\end{gathered}
$$

$$
\begin{gathered}
P V \\
\text { cal } / \text { mole }
\end{gathered}
$$$$
\begin{gathered}
E \\
\mathrm{cal} / \mathrm{mole}
\end{gathered}
$$

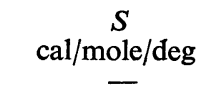

$$
-\overline{0,38}
$$$$
\underset{\mathrm{cal} / \mathrm{mole}}{L}
$$$$
-700
$$$$
-730
$$$$
-660
$$$$
-0,28
$$$$
-0,33
$$$$
-\overline{115}
$$

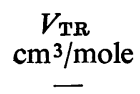

$\underset{\mathrm{kba}}{P_{\mathrm{TR}}}$

$3,59 \pm 0,16$

8,3

$$
\begin{array}{rl}
8,1 \pm 0,1 & 3,680 \pm 0,05 \\
8 \pm 0,16 & 3,560 \pm 0,02
\end{array}
$$

\section{TALBeAu IV}

Données thermodynamiques pour la transition de $\mathrm{RbBr}$ à $25^{\circ} \mathrm{C}$ [Thermodynamical data for the $\mathrm{RbBr}$ transition at $25^{\circ} \mathrm{C}$ ]

Pistorius

Weir et al.

Darnell, Mc Collum

Nos résultats
$2,19 \overline{ \pm} 0,8$

$$
1,371
$$$$
2,11 \pm 0,2
$$

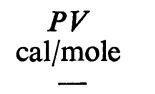

$\mathrm{cal} / \mathrm{mole}$

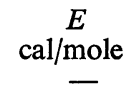

1/mole

$S$
$\mathrm{cal} / \mathrm{mole} / \mathrm{deg}$

$-\overline{0,35}$

$-700$

$-750$

$-642$

-680
-542

$-0,34$

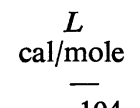

$-\overline{104}$

$-100$

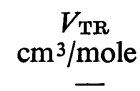

$P_{\text {TR }}$

$4,2 \pm 0,2$

6,61

$7,43 \pm 0,18$

$4,92 \pm 0,06$

$6,65 \pm 0,13$

\section{TALBEAU V}

\section{Données thermodynamiques pour la transition de $\mathrm{RbCl}$ à $25^{\circ} \mathrm{C}$}

[Thermodynamical data for the $\mathrm{RbCl}$ transition at $25^{\circ} \mathrm{C}$ ]

Pistorius

Weir et al.

Darnell, Mc Collum

Nos résultats

$$
\mathrm{d} P / \mathrm{d} T
$$
$\mathrm{kbar} /{ }^{\circ} \mathrm{C} \times 10^{3}$ $P V$
cal/mole
$E$
cal $/$ mole $\stackrel{S}{\mathrm{cal} / \mathrm{mole} / \mathrm{deg}}$ $-\overline{0,44}$ $\underset{\text { cal } / \text { mole }}{L}$ $-\overline{130}$

$$
2,8
$$$$
3,01
$$$$
3,38 \pm 0,4
$$
$-700$$$
-700
$$

$$
\text { - }
$$$$
-800
$$$$
-750
$$$$
-675
$$$$
-605
$$

$$
-0,42
$$$$
-0,49
$$

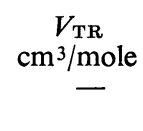

$\begin{gathered}P_{\mathrm{TR}} \\ \mathrm{kbar}\end{gathered}$
$5,28 \pm 0,44$

5,76

$-125$

$6,95 \pm 0,11$

$6,03 \pm 0,12$
4. Conclusions. - Cette étude a permis de déterminer les pressions des transitions polymorphiques d'halogénures du $\mathrm{Rb}$ en fonction de $T$ avec une précision voisine de $1 \%$. Ce résultat n'a pu être atteint que grâce à l'emploi d'une technique donnant accès à la zone d'indifférence ; ce qui implique presque obligatoirement un volume d'échantillon déjà important.

Malgré des impératifs d'encombrement rigoureux, car imposés par les dimensions internes de l'enceinte haute pression, et un fonctionnement en milieu liquide sous haute pression, le four que nous avons conçu nous a permis d'atteindre des températures de l'ordre de $450^{\circ} \mathrm{C}$. Bien que ces conditions expérimentales soient peu favorables à un isolement thermique de haute efficacité, la solution que nous avons apportée à ce problème s'est avérée très satisfaisante. De ce fait, les puissances nécessaires sont relativement modérées. Les performances de ce four, tributaires en grande partie de la tenue en température du liquide de confi- nement, semblent pouvoir être sensiblement étendues. En préalable, une étude systématique du comportement des liquides transmetteurs de la pression, en fonction de la température, paraît indispensable pour connaître les limites d'utilisation.

La gamme de températures que nous avons couverte a permis d'effectuer des recoupements avec les résultats assez disparates de la littérature. Ainsi, des comparaisons avec les résultats de Bridgman, limités à $100^{\circ} \mathrm{C}$, et de Pistorius, dans la gamme $20-200^{\circ}$, ont été faites. Il en est de même avec ceux de Darnell et Mc Collum, qui peu significatifs dans cette zone de température, ne nous paraissent pas devoir présenter une précision satisfaisante, compte tenu de la méthode utilisée, pour des températures inférieures à $400^{\circ} \mathrm{C}$. Cette confrontation montre clairement l'avantage que présente l'utilisation de la zone d'indifférence, en milieu hydrostatique, sur les méthodes précédemment employées. 
Par ailleurs, en l'absence de données suffisamment précises sur $d P / d T$, un certain doute existait quant à la validité des calculs que l'on pouvait faire des énergies de réseaux des halogénures précités. L'amélioration que nous avons pu apporter à la connaissance des pressions de transitions en fonction de $T$ permettent une évaluation plus sûre de diverses propriétés thermodynamiques et une approche plus précise des énergies mises en jeu.

\section{Bibliographie}

[1] Bridgman, P. W., The pressure transitions of the Rubidium halides, Z. Krist. allogr. 67 (1928) 363-376.

[2] Pistorius, C. W., Polymorphic transitions of the alkali chlorides at high pressures to $200^{\circ} \mathrm{C}, \mathrm{J}$. Phys. Chem. Solids, 25 (1964) 1477-1481.

[3] Pistorius, C. W., Polymorphic transitions of the alkali bromides and iodides at high pressures to $200^{\circ} \mathrm{C}$, J. Phys. Solids, 26 (1965) 1003-1011.

[4] Darnel, A. J. and Mc Collum, W. A., Thermodynamics of the $\mathrm{Fm} 3 \mathrm{~m}, \mathrm{Pm} 3 \mathrm{~m}$ transitions in the potassium and rubidium halides, J. Phys. Chem. Solids, 31 (1970) 805-815.

[5] Bridgman, P. W., The velocity of polymorphic changes between solids. Proc. Am. Acad. Sci. 52 (1916) 57-88.

[6] Lacam, A. and PeYronneau, J., Transitions hautes pres- sions. Zone d'indifférence des halogénures de Rubidium, Rev. Phys. Appl. 10 (1975) 295.

[7] Lacam, A. and Peyronneau, J., Transition polymorphique de $\mathrm{RbCl}$ sous haute pression. Influence des paramètres expérimentaux sur l'effet d'hystérésis, J. Physique, 34 (1973) 1047.

[8] Lacam, A. and Peyronneau, J., Transitions polymorphiques « ultra-rapides » induites par la pression, dans les halogénures alcalins, Rev. Phys. Appl. 10 (1975) 299.

[9] Arslanov, V. A., Vichnievsky, R., Vo Thanh, D., PeyRONNEAU, J. and LACAM, A., Détermination expérimentale de la conductivité thermique des liquides sous hautes pressions. Cas de l'huile de Silicone SI.550, (à paraître).

[10] Peyronneau, J., Thèse Paris 1973 non publiée. 